\title{
Setting up the HyDRA blind challenge for the microhydration of organic molecules
}

September 19, 2021

Taija L. Fischer ${ }^{a}$, Margarethe Bödecker ${ }^{a}$, Anne Zehnacker-Rentien ${ }^{b}$,

Ricardo A. Mata ${ }^{a}$, Martin A. Suhm ${ }^{a}$

${ }^{a}$ Institut für Physikalische Chemie, Universität Göttingen, Tammannstr. 6, 370r7 Göttingen, Germany; ${ }^{b}$ Institut des Sciences Moléculaires d'Orsay, Université Paris-Saclay, CNRS, 91405, Orsay, France

\begin{abstract}
The procedure leading to the first HyDRA blind challenge for the prediction of water donor stretching vibrations in monohydrates of organic molecules is described. A training set of 10 monohydrates with experimentally known and published water donor vibrations is presented and a test set of 10 monohydrates with unknown or unpublished water donor vibrational wavenumbers is described together with relevant background literature. The rules for data submissions from computational chemistry groups are outlined and the planned publication procedure after the end of the blind challenge is discussed.
\end{abstract}

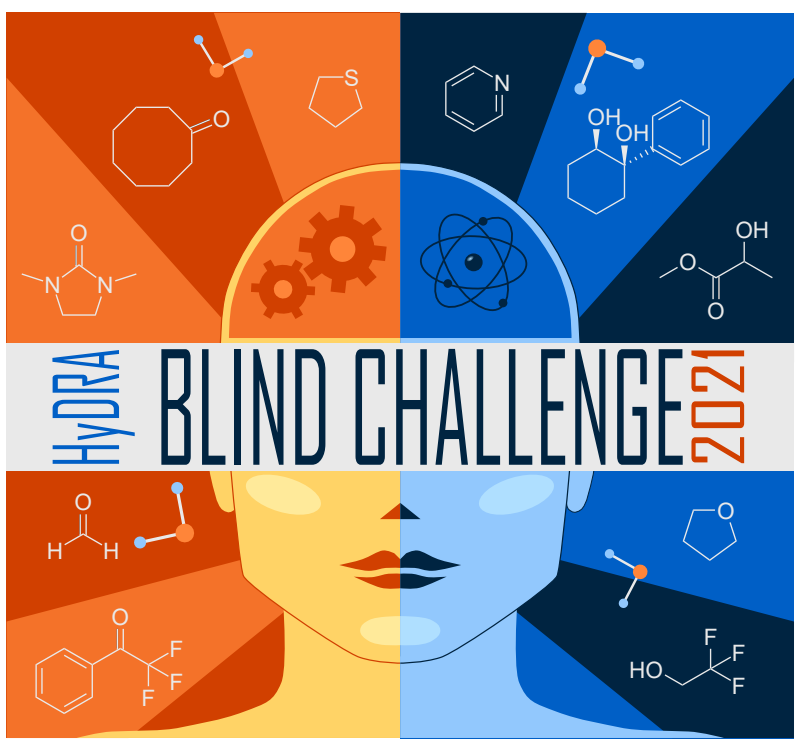

** please send all correspondence to M. A. Suhm (msuhm@gwdg.de) 


\section{Introduction}

The microhydration of organic matter ${ }^{1-3}$ deserves a systematic, unbiased comparison between theory and experiment ${ }^{4}$. The primary and most fundamental quantities to be benchmarked are probably structure and energy of cold, neutral 1:1 hydrate complexes prepared in supersonic jet expansions ${ }^{5}$. Any isomerism in the docking of individual water molecules to multifunctional organic molecules ${ }^{6}$ is of particular interest in this context, because theoretical input is most valuable and reliable for trends and comparisons ${ }^{7}$. This is where systematic model errors often tend to cancel for good reasons ${ }^{8}$, paving the way for helpful approximations. The properties of monoisomeric systems are valuable for a first calibration of absolute quantities, which are typically more challenging because they require highly accurate treatments of electronic structure and nuclear dynamics effects. It is rewarding that there are numerous rotational spectroscopy studies of monohydrates with more or less direct structural and sometimes relative energy information available in the literature ${ }^{5,9-11}$. These provide a lot of detail on the shallow potential energy landscapes of solvation phenomena.

A currently underexplored proxy to the hydrogen bond structure and energy of microhydrates is the wavenumber of the hydrogen-bonded $\mathrm{OH}$ stretching vibration $\tilde{\nu}_{\mathrm{OH}_{\mathrm{b}}}$ of any docking water serving as a hydrogen bond donor ${ }^{12}$. As a single number for a given monohydrate complex which can be derived from vibrational spectroscopy in a straightforward way, it offers a set of advantages over more demanding structural studies. For multifunctional substrates, linear IR and Raman spectroscopy can provide an immediate survey over competing hydrogen bond docking sites due to the simplicity and intensity of the vibrational fingerprint of the $\mathrm{OH}$ group ${ }^{13,14}$. Double resonance techniques allow for rigorous size and conformational distinction ${ }^{15}$, relaxation experiments can identify the most stable conformation ${ }^{16}$, and ${ }^{18} \mathrm{O}$ labelling of water can unambiguously discriminate from other hydride stretching modes in the vicinity ${ }^{17}$. The spectral position of the $\mathrm{OH}$ stretching vibration is very sensitive to the strength of the hydrogen bond, spanning about two orders of magnitude in relation to the typical spectral width of an isolated vibrational transition.

A theoretical model which successfully predicts such a single observable number like $\tilde{\nu}_{\mathrm{OH}_{\mathrm{b}}}$ is likely to initially get the right answer for the wrong reason, as multidimensional anharmonicity is not easy to model rigorously. This is particularly true if the experimental answer is known beforehand, due to a multitude of available and conceivable model variants and parameters. Therefore, large training sets, blind test components and iterative refinements are essential ingredients on the path towards systematic success for the modeling of $\mathrm{OH}$ stretching wavenumbers of monohydrates. Even a largely empirical model with a high success rate for a selected observable can be useful for the experimental gas phase cluster community. This is still close to the current status, where each observable and sometimes even research group has 
its favourite recipes (functionals, basis sets, scaling factors) to assist experiment. There may be more or less systematic correlations between the observed wavenumber shifts and experimental or computed proton affinities and gas phase basicities ${ }^{18-20}$ or calculated hydrogen bond lengths ${ }^{21}$, to name just a few possible links to be exploited. Ultimately, one must hope for a number of systematically successful models which connect theory and experiment on multiple observables, not only the vibrational wavenumber. Meaningful approximations such as elaborate scaling techniques ${ }^{22,23}$, local mode models ${ }^{24,25}$, parameterised harmonic DFT predictions ${ }^{26}$, polarisable force fields ${ }^{27}$ or hierarchical models building on high level treatments of the smallest systems or subgroups ${ }^{28}$ may be identified or we may even witness the victory of machine-learning approaches $^{29}$ towards such challenges.

While blind challenges have become popular in different chemical subcommunities such as protein $^{30}$ and crystal structure prediction ${ }^{31}$ or physicochemical data for drug molecules in solution $^{32-35}$, we are not aware of a blind challenge explicitly addressing vibrational spectroscopy data as a rigorous connecting point between theory and experiment. This may also be due to the fact that this connection relies on challenging electronic structure and vibrational anharmonicity issues at the same time. However, this is also subtly true for observables like molecular structure $^{36}$ and energy differences ${ }^{37}$, and therefore it appears timely to investigate a more bold case.

The HyDRA challenge is meant to kick off such a systematic approach to microhydration, with numerous conceivable follow-up options. HyDRA stands for Hydrate Donor Redshift Anticipation, where redshift refers to the wavenumber downshift of the donor $\mathrm{OH}$ vibration relative to the symmetric stretching fundamental of the water molecule ${ }^{38,39}$ at $3657 \mathrm{~cm}^{-1}$. The switch between absolute values and relative trends is thus trivial in this case, because it only involves adding or subtracting this experimental monomer value. In the present work, we use the sign convention that a positive downshift describes a lower wavenumber in the complex than in monomeric water. Here, we outline the construction of an initial training and test set for neutral organic monohydrates which was recently offered to theory groups ${ }^{40}$, connected with the invitation to participate in a blind challenge.

\section{Molecular systems}

\subsection{Experimental training set}

The first goal in this challenge was to identify a number of vibrationally well-characterised, diverse monohydrate complexes of small organic molecules in the existing experimental literature, for which the lowest energy structure involves water as the hydrogen bond donor and thus generates a characteristic donor $\mathrm{OH}$ stretching vibrational fundamental $\nu_{\mathrm{OH}_{\mathrm{b}}}$. For this purpose, 


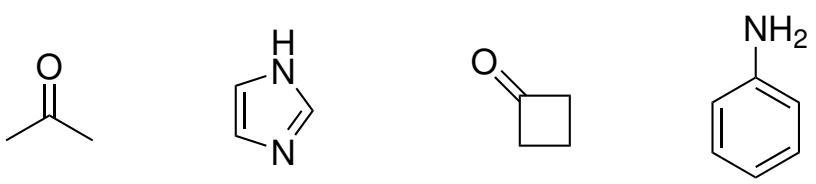

ACE: 67-64-1 IMZ: 288-32-4 CBU: 1191-95-3 ANL: 62-53-3

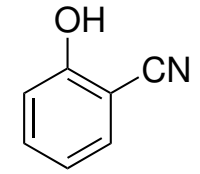

OCP: 611-20-1

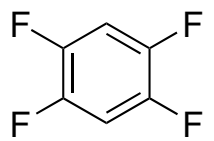

TFB: $327-54-8$

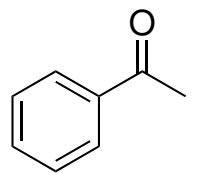

APH: 98-86-2

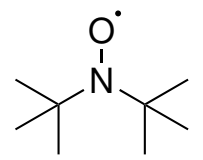

DBN: 2406-25-9

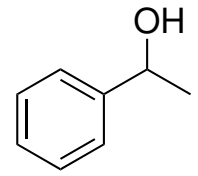

POH: 98-85-1

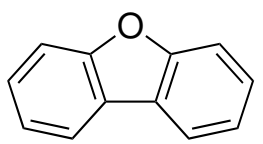

DBF: $132-64-9$

Figure 1: The structural formulas, abbreviations, and CAS registry numbers of the 10 systems selected for the HyDRA training set, sorted according to their electron number.

a call among vibrational spectroscopists was initiated early in 2021 and the suggestions were collected on a web site ${ }^{40}$, summarising the observed wavenumber $\tilde{\nu}_{\mathrm{OH}_{\mathrm{b}}}$, the organic species (with CAS number), the DOI of the relevant publication and the contact email of the submitter.

Further mandatory entries of every submission included the proposing researcher and institution (not necessarily from the group publishing the experimental data) as well as the spectroscopic detection, wavenumber calibration, and cooling techniques used. Concerning the observed wavenumber, the submitter had to estimate its accuracy, to predict the relevant docking site for the observed water molecule and to assess whether the monohydrate was the global minimum arrangement of the two complex partners.

Optional entries for further assessment and use of the submissions beyond the initial training included supporting researchers like coworkers and endorsing colleagues as well as spectroscopic data on the free (or otherwise engaged) $\mathrm{OH}$ stretching vibration of the hydrate water $\left(\tilde{\nu}_{\mathrm{OH}_{\mathrm{f}}}\right)$, the wavenumber and relative intensity of a potential resonance partner to $\nu_{\mathrm{OH}_{\mathrm{b}}}$ (possibly stealing intensity and shifting its band center), and any isotope substitution information or information on observed docking isomers.

Because it is essential for the challenge that the training set data are well secured, it was possible to list additional assignment measures concerning the organic molecule conformation, the docking site, and the size assignment to a monohydrate, as well as to comment on any other relevant issue.

Based on more than a dozen submissions from half a dozen countries over half a year, a small committee consisting of a PhD student, an undergraduate research student and two experimental spectroscopy group leaders made a selection of 10 systems to be recommended to 
the theory community for the training of their models. Among others, methanol ${ }^{14}$ was not included into the core training set because of its very large amplitude internal motion and a capped phenylalanine ${ }^{41}$ was not included because the global minimum hydrate structure presumably does not involve water as a hydrogen bond donor, although an energetically excited state does. Several ketones (cycloheptanone, cyclohexanone, 4,4-dimethylcyclohexanone, (-)-fenchone, 2-fluoroacetophenone, pinacolone, 3,3,5,5-tetramethylcyclohexanone) ${ }^{42,43}$ were not included to keep the balance between represented functional groups. The growing set of nonselected submissions is still made available, with the goal of a comprehensive curated data base on $\mathrm{OH}$ stretching wavenumbers of hydrate complexes in mind. Numerous interesting monohydrates are still missing in the data base, such as systems where the water induces a strong restructuring of the solute ${ }^{41}$ or very floppy ring systems ${ }^{44}$.

Table 1: Desirable properties for the training set members together with individual assessments whether they and their monohydrates fulfill them $(+)$ or not $(-)$.

\begin{tabular}{|c|c|c|c|c|c|c|c|c|c|c|}
\hline & $\mathrm{ACE}$ & $\mathrm{APH}$ & $\mathrm{ANL}$ & $\mathrm{CBU}$ & $\mathrm{DBF}$ & $\mathrm{DBN}$ & IMZ & $\mathrm{OCP}$ & $\mathrm{POH}$ & TFB \\
\hline $\begin{array}{l}\text { unique observed } \\
1: 1 \text { conformer? }\end{array}$ & + & - & + & + & + & + & + & + & + & + \\
\hline global minimum? & + & + & + & + & + & + & + & + & + & + \\
\hline isotope data? & + & - & - & - & - & - & + & - & - & - \\
\hline $\begin{array}{l}\text { Further experimental } \\
\text { references? }\end{array}$ & + & + & + & + & + & + & + & - & + & - \\
\hline $\mathrm{OH}_{\mathrm{f}}$ known? & + & + & + & + & + & + & - & + & + & + \\
\hline $\begin{array}{l}\text { potential b2lib- } \\
\text { resonance? }\end{array}$ & + & + & + & - & - & + & - & - & - & - \\
\hline less than $100 \mathrm{e}^{-}$? & + & + & + & + & + & + & + & + & + & + \\
\hline $\begin{array}{l}\text { lowest monomer- } \\
\text { vibration }>30 \mathrm{~cm}^{-1} \text { ? }\end{array}$ & - & - & + & + & + & + & + & + & + & + \\
\hline
\end{tabular}

The 10 systems selected for the HyDRA training set are shown in Figure 1 together with the three-letter acronym used in this work and with their CAS registry number. Each of them fulfills a number of desirable properties for this initial training process (Table 1). All are believed to have water acting as a hydrogen bond donor in the global minimum structure, all contain less than 100 electrons to facilitate accurate quantum-chemical modeling. All except APH (where IR spectroscopy was required to detect the second isomer ${ }^{42,45}$ ) have only one experimentally observed monohydrate conformation. For all except IMZ, the free $\mathrm{OH}$ stretching wavenumber of the solvating water is also known experimentally. All except for the methyl ketones ACE and APH with their low barrier methyl torsions are fairly stiff, with no monomer fundamental wavenumber significantly below $30 \mathrm{~cm}^{-1}$. For most monohydrates of the training set, there 
is more than one spectroscopic study in the literature, and in a few cases (ACE, IMZ) there are also isotope substitution experiments. In particular, many monohydrates (APH, ANL, CBU, DBF, IMZ) have been characterised structurally by rotational spectroscopy ${ }^{45-49}$. In two cases $\left(\mathrm{ACE}, \mathrm{APH}\right.$ ), there is a well-established vibrational b2lib resonance ${ }^{42}$ (a resonance of the water $\mathrm{OH}$ stretch with the bending overtone (b2) and a hydrogen bond librational (lib) mode) which makes these systems particularly interesting for advanced anharmonic models, but models not including such a resonance can also be applied, because we provide the deperturbed $\mathrm{OH}$ stretching wavenumber as well. In two other cases (ANL, DBN), there may be an analogous resonance, but this remains experimentally open and therefore the deperturbed value for $\mathrm{OH}_{\mathrm{b}}$ spans the experimentally observed main transition. To analyse such resonances, it is essential to avoid embedding of the monohydrates in matrices, because matrices may also induce site splittings which are difficult to distinguish from a resonance ${ }^{50}$.

In terms of experimental techniques, 5 monohydrates were characterised by UV/IR double resonance techniques (in different laboratories in France, Germany, Japan and Spain) and 6 by FTIR spectroscopy (in Germany), all in supersonic jet expansions. 6 compounds are aromatic, 1 is a radical, 3 are ketones, 2 are alcohols. The hydrogen bond acceptor atoms include O, F, $\mathrm{N}$ and $\pi$-systems.

Table 2: Training set of 10 acceptor molecules - abbreviations, CAS registry numbers (CAS RN) and published experimental $\mathrm{OH}_{\mathrm{b}}$-wavenumbers $\tilde{\nu}_{\exp }\left(\mathrm{OH}_{\mathrm{b}}\right)$ (with estimated uncertainty in parentheses) of the acceptor molecules in the monohydrates selected for the training set. In square brackets, estimated deperturbed $\mathrm{OH}_{\mathrm{b}}$ wavenumbers after removal of a three-quantum resonance are provided. These wavenumbers in brackets should be targeted by vibrational models which do not include such an anharmonic resonance. Where the resonance remains hypothetical, the deperturbed wavenumber uncertainty (in parentheses) spans the experimentally observed main transition wavenumber. Also provided are downshifts $\Delta_{\mathrm{OH}} / \mathrm{cm}^{-1}$ (positive, if the complex has a lower wavenumber) from the water monomer fundamental at $3657 \mathrm{~cm}^{-1}$.

\begin{tabular}{|c|c|c|c|c|}
\hline acceptor molecule & abbreviation & CAS RN & $\tilde{\nu}_{\text {exp }}\left(\mathrm{OH}_{\mathrm{b}}\right) / \mathrm{cm}^{-1}$ & $\Delta_{\mathrm{OH}} / \mathrm{cm}^{-1}$ \\
\hline Acetone $^{42}$ & $\mathrm{ACE}$ & $67-64-1$ & $3538(1)[3531(2)]$ & $119(1)[126(2)]$ \\
\hline Acetophenone ${ }^{42}$ & $\mathrm{APH}$ & $98-86-2$ & $3536(1)[3530(2)]$ & $121(1)[127(2)]$ \\
\hline Aniline $^{51}$ & ANL & $62-53-3$ & $3524(1)[3526(3)]$ & $133(1)[131(3)]$ \\
\hline Cyclobutanone ${ }^{42}$ & $\mathrm{CBU}$ & $1191-95-3$ & $3548(1)$ & $109(1)$ \\
\hline Dibenzofuran $^{48}$ & DBF & $132-64-9$ & $3623(1)$ & $34(1)$ \\
\hline Di-tert-butyl nitroxide ${ }^{52}$ & DBN & $2406-25-9$ & $3484(2)[3487(4)]$ & $173(2)[170(4)]$ \\
\hline Imidazole $^{17}$ & IMZ & $288-32-4$ & $3458(2)$ & $199(2)$ \\
\hline$o$-Cyanophenol ${ }^{53}$ & $\mathrm{OCP}$ & $611-20-1$ & $3595(2)$ & $62(2)$ \\
\hline 1-Phenylethanol ${ }^{54}$ & $\mathrm{POH}$ & $98-85-1$ & $3620(2)$ & $37(2)$ \\
\hline $1,2,4,5$-Tetrafluorobenzene ${ }^{55}$ & TFB & $327-54-8$ & $3647(1)$ & $10(1)$ \\
\hline
\end{tabular}

The target benchmark observables are summarised in Table 2. Either the absolute $\mathrm{OH}_{\mathrm{b}}$ - 
wavenumbers $\tilde{\nu}_{\text {exp }}\left(\mathrm{OH}_{\mathrm{b}}\right)$ of the hydrogen-bonding water or equivalently their downshifts $\Delta_{\mathrm{OH}}$ from the isolated water symmetric stretching fundamental can be targeted. For models including full anharmonicity and thus the postulated Darling-Dennison 1-3 resonance for four systems, the most intense $\mathrm{OH}_{\mathrm{b}}$ transition can be addressed directly. For models which do not include such resonances, it may be better to address the values in square brackets, which attempt to correct for this anharmonic perturbation with a coupling matrix element on the order of $10 \mathrm{~cm}^{-1}$ and involve a larger error bar due to the correction uncertainty. For imidazole it is worth mentioning that most techniques detect both the $\mathrm{NH}$ - and $\mathrm{OH}$-bonded isomers ${ }^{49,56}$, whereas theory and a slit jet expansion study ${ }^{17}$ indicate a clear energetical preference for the $\mathrm{OH}$-bonded isomer which is of interest in the context of this challenge.

\subsection{Experimental test set}

For the experimental test set of vibrationally uncharacterised monohydrates, a multifacetted approach was chosen. Because their experimental data have to be secured and kept secret until the end of the blind challenge, some of the considered systems were already experimentally characterised in the research groups forming the selection committee, but not yet published. Others were at least pre-explored to increase the likelihood of a successful assignment during the runtime of the blind challenge. Furthermore, an informal call to microwave spectroscopy groups was made to collect suggestions for systems which had already been characterised structurally, to minimise the risk that a subsequent vibrational characterisation meets unexpected difficulties. This approach led to a preselection of 15 monohydrate proposals, for which there is no gas phase vibrational spectroscopy record in the literature. From those, the final selection of 10 official test systems was made one month after the publication of the training set, by the same selection committee. These 10 target systems for the blind challenge are summarised in Figure 2 (ordered by number of electrons) and Table 3 (in alphabetical sequence).

Each target system, ordered by increasing number of electrons and thus computational complexity, will be briefly discussed in the following to introduce the most relevant literature sources for structural and other information.

For FAH as the simplest molecule in the test set, it is long established by theory ${ }^{74-76}$ and experiment ${ }^{62}$ that water coordinates asymmetrically. The $\mathrm{OH}$ stretching vibration which is of interest in this challenge has been determined in matrix isolation ${ }^{61,77}$, but to the best of our knowledge not in the gas phase without environmental influence. In cryogenic matrices, split signals were found $\left(3580,3585 \mathrm{~cm}^{-1}\right.$ in neon and argon, $3573,3578 \mathrm{~cm}^{-1}$ in a nitrogen matrix). These can give a first orientation, but the vacuum-isolated transition may be located above, below or in this range and the splitting may be lifted if it is caused by matrix interaction. Whether the complex is planar or quasiplanar is difficult to determine with the available experimental data ${ }^{62}$, but we expect to provide a single experimental vacuum-isolated water $\mathrm{OH}_{\mathrm{b}}$ stretching 


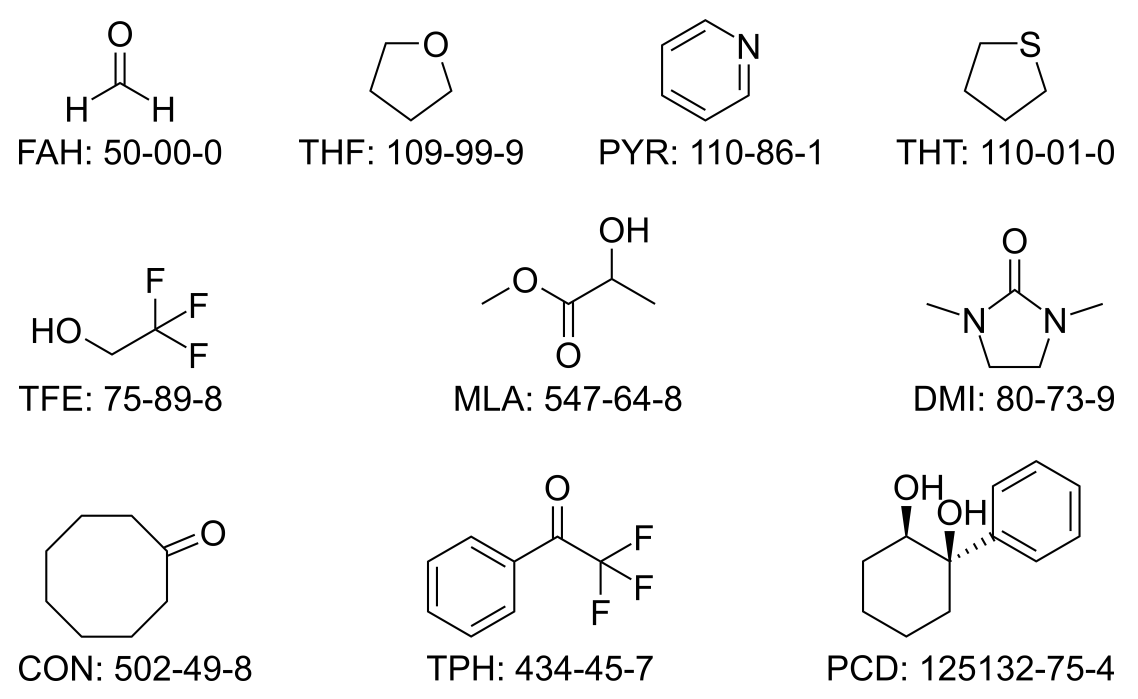

Figure 2: The structural formulas, abbreviations, and CAS registry numbers of the 10 systems selected for the HyDRA test set.

wavenumber to be predicted by the different models.

Because of its relevance for clathrate formation, the $\mathrm{OH}$ stretching spectrum of the THF monohydrate has been obtained in $\mathrm{CCl}_{4}$ solution ${ }^{78}$ at $268 \mathrm{~K}$. A broad (about $80 \mathrm{~cm}^{-1} \mathrm{FWHM}$ ) hydrogen-bonded $\mathrm{OH}$ stretching fundamental was located near $3450 \mathrm{~cm}^{-1}$ at high dilution. Due to thermal and solvent effects, this absolute value is not suitable for the direct comparison to environment-free ground state calculations. In a theoretical study from 2005, it was concluded that the complex has never been observed in spectroscopic experiments ${ }^{69}$. With respect to gas phase investigations, we found no evidence to revise this statement. In particular, there appears to be no rotational spectroscopy characterisation of the monohydrate, but one can expect one or two isomers, due to the complex pseudorotational dynamics of the monomer ${ }^{68,79}$.

The PYR monohydrate is a model system for photoinduced homolytic water splitting ${ }^{80}$. Its structure is quite flexible, as evidenced by rotational spectroscopy ${ }^{67}$. It includes a tunneling motion with an inverted H/D isotope effect, but this is not directly relevant for the vibrational transition at low resolution. There is a room temperature gas phase value for the hydrogenbonded OH stretching mode in PYR monohydrate ${ }^{81}$, at $3480 \mathrm{~cm}^{-1}$. However, for such a soft and flexible hydrogen bond, it is likely that the cold transition obtainable in a jet expansion occurs at significantly lower wavenumber. While ultracold helium droplets should exhibit much smaller, but still significant ${ }^{17,82}$ environmental shifts than a room temperature gas phase measurement, a corresponding recent study ${ }^{66}$ does not address the relevant spectral range and isotope composition.

In contrast to the THF situation, the monohydrate of THT has been structurally characterised in much detail ${ }^{70}$, also with respect to the water orientation relative to the ring. We are not aware of a complementary vibrational gas phase study and thus consider this to be a valuable diversification of the test set, also in preparation for more challenging thio compounds ${ }^{83}$. 
Table 3: Test set of 10 acceptor molecules - abbreviations, CAS registry numbers (CAS RN) and expected minimum number of $\mathrm{OH}$ stretching signals in the most stable monohydrate $n(\mathrm{OH})$, among which the hydrogen-bonded water $\mathrm{OH}_{\mathrm{b}}$ wavenumber $\tilde{\nu}_{\exp }\left(\mathrm{OH}_{\mathrm{b}}\right)$ (or its downshift $\Delta_{\mathrm{OH}} / \mathrm{cm}^{-1}$ from the water monomer fundamental at $3657 \mathrm{~cm}^{-1}$ ) should be predicted. If a three-quantum resonance is predicted, estimated deperturbed water $\mathrm{OH}_{\mathrm{b}}$ wavenumbers after removal of this resonance may also be provided. For $n(\mathrm{OH})>2$ (alcohols), we recommend that the theory groups report all $\mathrm{OH}$ stretching fundamentals, because there may be mode mixing between the hydrogen-bonded alcoholic and water $\mathrm{OH}$ bonds. Alternatively, we recommend to report the mode which shows the strongest downshift when $\mathrm{H}_{2}{ }^{16} \mathrm{O}$ is replaced by $\mathrm{H}_{2}{ }^{18} \mathrm{O}$, because it carries the strongest water stretching character.

\begin{tabular}{|c|c|c|c|}
\hline acceptor molecule & abbreviation & CAS RN & $n(\mathrm{OH})$ \\
\hline Cyclooctanone $^{57,58}$ & $\mathrm{CON}$ & $502-49-8$ & 2 \\
\hline 1,3-Dimethyl-2-imidazolidinone ${ }^{59,60}$ & DMI & $80-73-9$ & 2 \\
\hline Formaldehyde ${ }^{61,62}$ & $\mathrm{FAH}$ & $50-00-0$ & 2 \\
\hline Methyl lactate 63,64 & MLA & $547-64-8$ & 3 \\
\hline 1-Phenylcyclohexane-cis-1,2-diol ${ }^{65}$ & PCD & $125132-75-4$ & 4 \\
\hline Pyridine 66,67 & PYR & $110-86-1$ & 2 \\
\hline Tetrahydrofuran 68,69 & $\mathrm{THF}$ & 109-99-9 & 2 \\
\hline Tetrahydrothiophene ${ }^{70}$ & THT & $110-01-0$ & 2 \\
\hline 2,2,2-Trifluoroacetophenone ${ }^{71}$ & $\mathrm{TPH}$ & $434-45-7$ & 2 \\
\hline 2,2,2-Trifluoroethan-1-ol ${ }^{72,73}$ & TFE & $75-89-8$ & 3 \\
\hline
\end{tabular}

In TFE, as in many fluorinated alcohols ${ }^{72}$, the first solvating water acts primarily as a hydrogen bond acceptor, but the symmetric stretching mode is only subtly shifted from the water monomer value by this interaction and by an additional donor contact to the $\mathrm{CF}_{3}$ group. This provides valuable benchmark information for small complexation shifts, but the IR activity of such weakly perturbed symmetric $\mathrm{OH}$ stretching motions in water molecules is low. Therefore, Raman spectroscopy ${ }^{14}$ is expected to provide key vibrational information in this case. Structurally, the preference of the monohydrate for an insertion complex is well established ${ }^{73}$ and the related, structurally more diverse case of hexafluoroisopropanol is also well studied ${ }^{84}$. By including the simplest model of a peptide co-solvent ${ }^{85}$ monohydrate into the vibrational target systems, the characterisation of their unusual biochemical properties can be supported ${ }^{86}$.

For MLA with its weak internal hydrogen bond between the hydroxy group and (preferentially) the carbonyl group, a solvent water has different options to coordinate. It can insert into the internal hydrogen bond, or add to either end, or even to the ester oxygen. Due to the activation barrier for the first process, jet experiments have to make sure that insertion is not kinetically hindered ${ }^{87}$. This is important, because theory predicts insertion to be most stable. In this context, it is essential that there is experimental microwave evidence for the inserted structure ${ }^{63}$, in which the solute also directs the dangling water hydrogen to one side. 
The competition from lactate conformations with the carbonyl group pointing away from the hydroxy group is intense in aqueous solution ${ }^{64}$, but not yet in the monohydrate. In contrast to jet studies of the methanol complex ${ }^{63}$ and matrix studies of the ammonia complex ${ }^{88}$, we are not aware of matrix isolation or jet studies of the $\mathrm{OH}$ stretching vibration of the monohydrate.

For the urea derivative DMI, a solution study ${ }^{59}$ has revealed broad absorption maxima of the monohydrate in 1,2-dichloroethane $\left(3431 \mathrm{~cm}^{-1}\right)$ and $\mathrm{CCl}_{4}\left(3460 \mathrm{~cm}^{-1}\right)$ solution, downshifted by 162 and $155 \mathrm{~cm}^{-1}$, respectively, from the monomer symmetric $\mathrm{OH}$ stretch of water in the same solution. We are not aware of structural data for the monohydrate in the gas phase, but there is evidence for involvement of the $\mathrm{C}=\mathrm{O}$ group in the hydrogen bond ${ }^{59}$ and the DMI monomer has been characterised by rotational spectroscopy ${ }^{60}$.

Monomeric CON has been structurally characterised by microwave jet spectroscopy and shown to exist in a dominant boat-chair conformation with non-equivalent lone pairs of the oxygen ${ }^{57}$. The energy difference to the next conformation is so large that monohydration is unlikely to switch the energy sequence. Therefore up to two isomers of the monohydrate are expected $^{58}$ and the more stable one is of interest in the present challenge. As the energy balance between the two docking isomers could be subtle, we recommend to calculate both and to provide at least the donor $\mathrm{OH}$ stretching wavenumber of the more stable one. We are not aware of any vibrational study of the monohydrate of CON.

$\mathrm{TPH}$ is an unsymmetric ketone, in which the water molecule is strongly directed to one of the $\mathrm{C}=\mathrm{O}$ lone electron pairs. Its monohydrate was recently accurately characterised by rotational spectroscopy and theory ${ }^{71}$ but we are not aware of a published gas phase vibrational study. Comparison to the well-studied non-fluorinated parent compound APH from the training set will reveal the influence of fluorination through space and through several bonds.

PCD as the most complex member of the test set is of stereochemical and hydrogen bond topological interest. Note that the two OH groups are cis-configured, whereas the absolute chirality is not relevant for the monohydrate. The diol forms two weak, but cooperative hydrogen bonds $(\mathrm{OH}-\mathrm{OH}$ and $\mathrm{OH}-\pi)$ which give rise to separate $\mathrm{OH}$ stretching transitions in $\mathrm{CCl}_{4}$ solution $^{65}$. The water in the monohydrate has several options to attach or insert into this hydrogen bond chain and the most stable conformation is sought. In this particular case, we recommend an extensive search among the possible conformations to identify the global minimum conformation. It will be characterised by IR/UV spectroscopy and isotope labelling. We are not aware of previous gas phase work on this system.

Figure 3 correlates the number of vibrational degrees of freedom $3 N_{n}-6$ with the number of electrons $n_{e}$ of the solutes for the training and test sets. The sets span an order of magnitude in each quantity and deviate significantly from a linear relationship, with some systems being more challenging for the nuclear dynamics treatment and others for the electronic structure treatment. Two of the test set members (FAH, PCD) represent an extrapolation relative to 


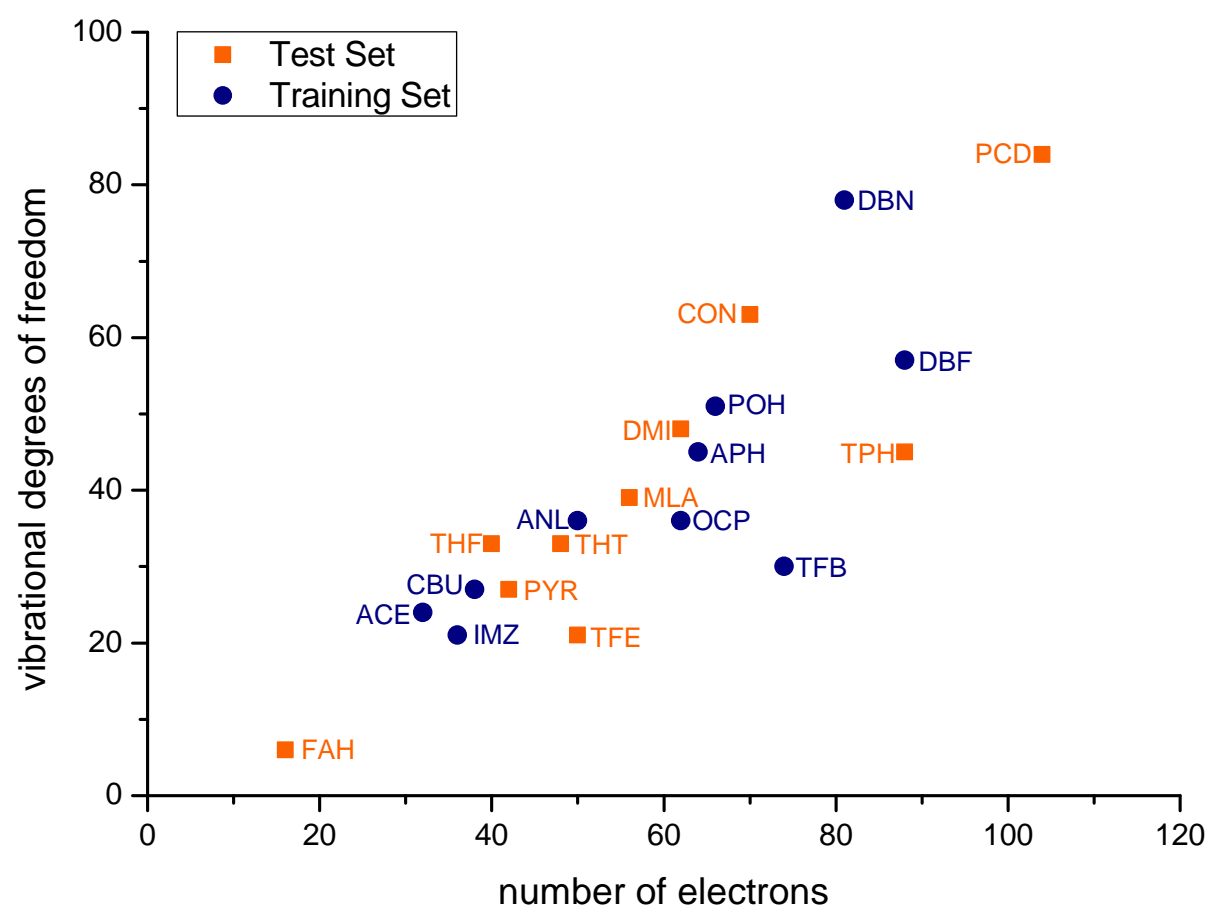

Figure 3: Vibrational degrees of freedom $3 N_{n}-6$ of the training (blue) and test (orange) solute molecules for which monohydrates are investigated as a function of their number of electrons $n_{e}$.

the training set, whereas the others tend to fill gaps in terms of electron and nuclear degrees of freedom.

Acceptors which were considered as suitable candidates for the test set, but are postponed for later investigation to maximise its diversity and to reduce its conformational complexity, include acetaldehyde (AAH: 75-07-0), 4'-fluoroacetophenone (FPH: 403-42-9), hexafluoroisopropanol (HFP: 920-66-1) ${ }^{84}$, N,N-dimethylformamide (DMF: 68-12-2) and 2,2,4,4-tetramethylpentan-3one (TPO: 815-24-7). Other candidates which require further testing or turned out to be less suitable at this stage include benzaldehyde, 4-fluorobenzaldehyde, furan, thiophene, propylene oxide, and 4-methoxy-2,2,6,6-tetramethyl-1-piperidinyloxy. Further interesting candidates which were suggested by microwave groups and will have to be further analysed in terms of suitability include propargyl alcohol ${ }^{89}$, furfuryl alcohol, and furfuryl mercaptane ${ }^{90}$ as well as thenyl alcohol and thenyl mercaptane ${ }^{83}$. 


\section{Experimental methods}

\subsection{Description of experimental procedures}

All experimental data of this blind challenge refer to translationally and rotationally cold molecules $(T<20 \mathrm{~K})$ without any significant environment, but with some residual vibrational excitation in soft modes from the initial thermal population. There is the possibility of conformational trapping behind barriers, which may partially avoid relaxation to the global minimum structure. This is the typical situation in adiabatic carrier gas expansion experiments into vacuum, either free ${ }^{91}$ or skimmed to form a molecular beam ${ }^{92}$, with a starting temperature of $300 \mathrm{~K}$ or somewhat higher for low-volatility compounds. The specific monohydrates are chosen to minimise the risk of significant conformational trapping in most cases. The trapping can be intermolecular, if two or more hydrogen bond formation sites are available in the solute molecule. In such a case, cold monohydrates with water docking on the less favourable site cannot quantitatively interconvert into the most favourable site on the timescale of the experiment in all cases. This is particularly relevant for the three alcohols in the test set, where water can attach to any lone pair or insert into a pre-existing hydrogen bond of the solute ${ }^{87}$. The insertion is typically an activated process and if the activation energy significantly exceeds $5 \mathrm{~kJ} / \mathrm{mol}$, it may not happen sufficiently fast under the low collision rates of the supersonic expansion. The trapping can also be of intramolecular nature, if the solute exists in different conformations with higher barriers. For ring puckering modes, the barriers are not always sufficiently low for efficient funneling into the vibrational ground state. For intramolecular hydrogen bonds, the solutes are chosen such that relaxation into the global minimum structure was typically proven experimentally, or is at least likely. Constitutional relaxation is evidently not considered. Thus, the decomposition of formaldehyde into $\mathrm{CO}$ and $\mathrm{H}_{2}$ is disregarded for the global minimum criterion, although it is exothermic by about $9 \mathrm{~kJ} \mathrm{~mol}^{-1}$ at low temperature ${ }^{93}$. The solutes always remain in the constitution given in Figure 2.

The spectroscopic methods used to probe the monohydrates may be distinguished into action and linear techniques. In action techniques, the effect of the photons on the monohydrates is probed, e.g. ionisation, dissociation or fluorescence. In linear techniques, the effect of the monohydrates on the photons is probed, e.g. direct infrared absorption ${ }^{13}$ or inelastic (Raman) scattering ${ }^{85}$. Action techniques often offer much higher sensitivity, they can be size-selective and conformationally selective ${ }^{15,94-96}$.

\subsection{Challenges and experimental difficulties}

Linear techniques probe all the molecular systems present in the expansion, based on their photon absorption or scattering cross sections. They can usually identify size (e.g. hydration 
number) by variation of the expansion conditions and by following the signal evolution, at least for 1:1 complexes if those do not spectrally overlap with other cluster compositions. Conformations can only be distinguished if they differ in their spectral fingerprint, sometimes with the help of isotope substitution. There is a less liberal molecular size limit for linear techniques due to the decreasing vapour pressure and the limited sensitivity. Action techniques may in turn suffer from unwanted competing processes such as fragmentation and may still be problematic for many non-aromatic systems and even some aromatic systems with fast processes in the electronically excited state ${ }^{97,98}$. The best is always a combination of both techniques, such as in the case of $\mathrm{POH}$ from the training set. This is, however, unrealistic on the time scale of this challenge and for the choice of molecules. It is anticipated that one member of the test set (PCD) will only be characterised by an action technique (IR/UV double resonance), whereas the others will be mostly accessed by direct infrared absorption. For the latter, a recent instrumental development ${ }^{99}$ is very helpful for monohydrates, because its gas recycling allows for the use of expensive isotopologues, solutes and carrier gases. In some cases, where the water vibration of interest has a low infrared intensity or may be distributed over several $\mathrm{OH}$ stretching modes in the complex, linear Raman spectroscopy ${ }^{14,85}$ can provide the required information.

Aggravated by the partial double-blind character of the challenge, it can not be strictly ruled out that a training or test set member has to be removed completely from the challenge, because experiments or their interpretations reveal a major problem. Such problems may include the discovery of new monohydrate or even monomer conformations which complicate an unambiguous assignment ${ }^{37}$, issues with the cluster size assignment, experimental downtimes due to instrument failure, commercial availability bottlenecks, etc.. However, we expect that such problems do not exceed a $10 \%$ threshold and thus do not significantly narrow the significance of the challenge.

\section{Challenge procedure}

\subsection{Theory group involvement}

Theory groups and other researchers interested in the systematic prediction of $\mathrm{OH}$ stretching wavenumbers for monohydrates were alerted about this blind challenge via news groups, conferences, and direct contact, together with the training set of 10 organic solvates. They were given the possibility to register for the challenge, to be alerted immediately upon the start of the actual competition with the announcement of the test set. Registrations up to the submission deadline are accepted. A unique entry code is provided to each participant in order to allow them later to review their own submitted data.

Although it does not stand as a strict rule, the calculations for all molecules in both sets 
(test and training) should be carried out as consistently as possible. This would mean that the level of theory at all different stages (conformer search, optimisation, calculation of frequencies, etc...) should be the same. The same also applies to non-structure based methods. For example, if a regression is used, the same model should be applied to all systems. However, it is expectable that some parameters of the protocol might change. For example, in some systems conformational searches might be skipped altogether, or carry different parameters due to the range of molecular sizes. It is also possible that some geometry optimisation criteria will be adapted. These computational details are expected to be provided at the end of the challenge. Assistance to all the participating groups is provided throughout the challenge duration, strictly avoiding any direct contact between the theory and the experimental groups, in the true spirit of a double-blind challenge.

One cannot rule out that we have overlooked published cold gas phase vibrational work on a system in the test set, or that such work appears on the scientific record before the experimental HyDRA data are released after the end of the challenge. This could be due to activities of other experimental groups not involved in the challenge, or due to data leakage from our experimental groups despite substantial effort to keep the results confidential among the contributing experimentalists. In such a case, the affected test set member will be shifted to training set status, because one cannot rule out that some model-proposing participants had a knowledge advantage over others. The goal is clearly to keep the blind character as unquestionable as possible ${ }^{32}$.

\subsection{Mandatory data submission}

The mandatory part of the submissions for this blind challenge will be compact. For each of the training and test set members, a single predicted wavenumber for the hydrogen-bonded water $\mathrm{OH}$ stretching mode in the most stable monohydrate in $\mathrm{cm}^{-1}$ must be provided in a standardised online form. We strongly recommend to provide together with this number an error estimate. This can be based on an actual statistical analysis (e.g., from the errors observed in the training systems) or even from personal convictions. Each participant group has the freedom to select which systems to compute (and ultimately submit data). In other words, all submissions will be accepted even if incomplete.

This compact table of up to 20 numbers (plus uncertainties) must be accompanied by a detailed description of the rational prediction method employed, to be included as a separate, autonomous file in the supplementary information of the planned publication. The description must contain all the details required for the reproduction of the results, such as the employed methods and program packages, with suitable references. Scaling parameters, employed atomic masses and any other parameters and computational keywords required for reproduction must be provided. This document will be in the full responsibility of the submitting authors and 
should include all the names and affiliations of the contributing co-authors as well as the role of each co-author in the submission. The use of ORCID ids is strongly recommended.

\subsection{Optional data submission}

Optional parts of the submission can be, in the sequence of decreasing importance for the goals of this blind challenge, and in free format to be included in the supplementary information:

a) A compact table of possible resonance partners of the hydrogen-bonded $\mathrm{OH}$ stretching mode of water, where predicted. This should include the wavenumber of the perturber and its fractional share of the intensity (square of the zero order $\mathrm{OH}$ stretching wavefunction), and for control purposes also the fractional share of the $\mathrm{OH}$ stretching wavenumber of the perturbed $\mathrm{OH}$ stretching state. From this and the mandatory submission of the predicted $\mathrm{OH}$ stretching wavenumber, a coupling matrix element can be obtained in the 2-level dark perturber approximation, see ${ }^{42}$. Such information will only be possible if anharmonic calculations or coupling models are included. For other submissions, the submitted $\mathrm{OH}$ stretching wavenumber will be taken as the deperturbed zero order $\mathrm{OH}$ stretch which is obtained from experiment in such a 2-level deperturbation, in the few cases where such a resonance is observed.

b) A table of any other $\mathrm{OH}$ stretching wavenumbers of the monohydrate, in particular the free $\mathrm{OH}$ stretch of the water and any other $\mathrm{OH}$ stretching mode in the acceptor molecule. If strong mixing of the $\mathrm{OH}$ oscillators is predicted, the mode with the strongest hydrogen-bonded water character should be submitted in the mandatory part and all the others in this part. One way to identify the mode with dominant water vibration character is to calculate the spectrum for $\mathrm{H}_{2}{ }^{18} \mathrm{O}$. The $\mathrm{OH}$ stretching mode with the strongest isotope downshift is the one of interest.

c) IR intensities and Raman scattering activities for all the $\mathrm{OH}$ stretching modes of the monohydrate, where available, preferably in $\mathrm{km} / \mathrm{mol}$ and in $\AA^{4} / \mathrm{u}$, including the depolarisation ratio for polarised light.

d) Any spectral information on isotopologues which may be useful for the experimental analysis and for follow-up work (D, $\left.{ }^{18} \mathrm{O}\right)$, always specifying the exact employed atomic masses. We recommend to always use the explicit isotopic masses for best method comparison ${ }^{100}$. Some programs use masses averaged over natural isotopic composition by default. This may have relatively small consequences for the current low resolution vibrational spectra and systems (still up to $5 \mathrm{~cm}^{-1}$ for vibrations involving sulfur due to a ratio of 1.003 between the natural atomic mass and the main isotope mass), but is clearly detrimental when comparing to high resolution (rotational) spectroscopy in the future.

e) Relative energy (in kJ/mol, excluding and including zero-point vibrational energy corrections) and spectral properties of any higher-lying conformations of the monohydrates which were investigated, within a range of $3-5 \mathrm{~kJ} / \mathrm{mol}$ of the global minimum structure. Here, global is defined under the restriction that the chemical bond connectivity remains the same, so rearranged 
bonding patterns like geminal diols as structural isomers of noncovalent ketone monohydrates ${ }^{93}$ are not considered. Geometries, when available, may be provided.

After the official submission deadline for model contributions, there will be a limited time window for the correction of submissions. Once this window is closed, the raw experimental data will also be made publicly available on a data repository, such that the challenge is closed on both ends.

\subsection{Evaluation of the blind challenge}

The submitted $\mathrm{OH}$ stretching data will be carefully evaluated and compared with the experimental values in different ways. Correlation plots for training, test and combined sets will be produced and analysed for all complete submissions, with a possible focus on which set is predicted better. Where several incomplete submissions focus on the most simple systems, which is to be expected for high-level theoretical treatments, analysis of such subsets with benchmark character will be attempted.

\subsection{Publication of the results}

The results will be published in a relatively compact many-author manuscript with extensive supplementary information in the responsibility of separate experimental and computational author subgroups, planned to be part of a PCCP themed collection on benchmarking in 2022. The final selection of the methods and results included will be subject to different criteria including diversity of methods, sophistication of the approach and total number of 'coincident' submissions. Our expectation is, however, that all submissions will be considered. The submission of the data does not change the ownership of the data, the latter remains exclusive to the participating group. Any participants whose results are not included are still welcome to submit their work independently. Participants selected to participate in the joint paper are expected to respond to two rounds of manuscript reviews and to remove inconsistencies and gaps. Failure to reply within a two-week period effectively confirming co-authoring could result in exclusion from the joint publication. The publication should be viewed as the starting point for numerous follow-up activities by the individual groups, such as completing or improving their predictions in a non-blind fashion. Groups which did not meet the submission deadline are invited to present their results independently as well. Some experimental assignments may have to be revisited in the light of systematically conflicting predictions. Optional data provided by the computational approaches may help to better understand the spectra. The double-sided theory-experiment interplay will continue, as usual in the field. All these activities profit from a fast publication of the primary blind challenge results. 


\subsection{Post-publication activities}

After publication of the results of the HyDRA blind challenge, the training and test sets can be extended to more strongly and even more weakly bound monohydrates, to charged systems (where more powerful experimental techniques are available ${ }^{101-103}$ ), to dihydrates, to isotope effects, to other vibrations, or to further observables such as structures and relative energies. These extensions may be targeted by different groups and will hopefully lead to a continuous refinement of the theoretical modelling of the microhydration of organic matter, identifying the most powerful and universal models in the field.

There are indeed many interesting monohydrates which were not considered for the present challenge for various reasons. This includes systems where the global minimum structure is hidden behind a large monomer isomerisation barrier ${ }^{104}$, and some systems which are closely related to members of the training or test set ${ }^{42,105}$. It also includes the numerous systems where the global minimum structure involves water as a pure acceptor. In terms of acceptor elements beyond $\mathrm{O}$ and $\mathrm{N}$, there are many extension possibilities towards $\mathrm{S}^{83,106,107}$ which remain to be explored. For the structurally well characterised acid hydrates ${ }^{108,109}$, one may expect complex vibrational dynamics whenever the $\mathrm{OH}$ stretching excitation comes close to degenerate proton transfer barriers.

\section{Conclusions}

We have described a first systematic blind challenge initiative in the field of hydrogen bondinduced shifts in solvating water molecules. It consists of a training set of 10 literature-known 1:1 hydrate complexes spanning a downshift window of nearly $200 \mathrm{~cm}^{-1} .10$ vibrationally unassigned monohydrates are proposed for the actual blind test and promised to be experimentally characterised during the runtime of the challenge. The combined experimental results and predictions will be jointly published to identify the most promising empirical, semiempirical and ab-initio models in the field. Several options for the extension of this initial challenge in chemical and spectral space are proposed.

We see this direct match between electronic structure theory and vibrational spectroscopy as an important complement to more traditional and well-established blind challenges in the field of protein ${ }^{110}$ or crystal structure ${ }^{111}$ prediction, where subtle energy differences also relate to zero point or thermal vibrational energy and thus anharmonicity treatments, besides getting the potential energy hypersurfaces right. This is even more obvious for the field of room temperature hydration ${ }^{112}$, where an atomistic method which systematically predicts correct free energy differences for a good reason is likely to predict reliable vibrational shifts of monohydrates as well. However, there is a long path of convergence between rigorously atomistic and other, more empirical or approximate or machine-learning models which are more easily extendable 
to macroscopic samples. Clearly, there is plenty of room in between. Blind challenges may be among the most objective procedures to find out where we stand.

\section{Author contributions}

T.L.F.: Data curation, Formal analysis, Investigation, Visualization, Writing - review \& editing; M.B.: Data curation, Investigation, Visualization, Writing - review \& editing; A.Z.: Investigation, Methodology, Validation, Writing - review \& editing ; R.A.M.: Conceptualization, Funding acquisition, Methodology, Project administration, Resources, Software, Writing - review \& editing ; M.A.S.: Conceptualization, Formal Analysis, Funding acquisition, Investigation, Methodology, Project administration, Resources, Supervision, Validation, Writing - original draft, Writing - review \& editing

\section{ORCID}

T.L.F. 0000-0003-2050-3628; M.B. 0000-0002-5883-3801; A.Z. 0000-0001-5540-0667; R.A.M. 0000-0002-2720-3364; M.A.S. 0000-0001-8841-7705;

\section{Acknowledgements}

We thank colleagues who have made helpful suggestions for the training and test sets, namely E. Arunan, D. Bernhard, J. Fernandez, A. Fujii, E. Gloaguen, A. Lesarri, M. Mons, D. Obenchain, M. Sanz, K. Schwing, R. Wugt Larsen, Y. Xu, J. Zischang. This work was funded by the Deutsche Forschungsgemeinschaft (DFG, German Research Foundation) - 389479699/GRK2455.

\section{Supporting information}

The supporting information contains short profiles for the 10 training set members to help contributors in the computational treatment and in the judgement of its feasibility and correctness.

\section{References}

[1] Felker, P. M.; Zewail, A. H. Stepwise solvation of molecules as studies by picosecond-jet spectroscopy: dynamics and spectra. Chemical Physics Letters 1983, 94, 454-460.

[2] Knochenmuss, R. D.; Smith, D. E. Time and internal energy dependent fluorescence spectra of naphthol-water clusters. The Journal of Chemical Physics 1994, 101, 73277336.

[3] Bombach, R.; Honegger, E.; Leutwyler, S. Solute-solvent interactions in microhydrate clusters: Carbazole-(H2O) n. Chemical Physics Letters 1985, 118, 449-454. 
[4] Puzzarini, C.; Bloino, J.; Tasinato, N.; Barone, V. Accuracy and interpretability: the devil and the holy grail. New routes across old boundaries in computational spectroscopy. Chemical Reviews 2019, 119, 8131-8191.

[5] Becucci, M.; Melandri, S. High-resolution spectroscopic studies of complexes formed by medium-size organic molecules. Chemical Reviews 2016, 116, 5014-5037.

[6] Mons, M.; Dimicoli, I.; Tardivel, B.; Piuzzi, F.; Robertson, E. G.; Simons, J. P. Energetics of the gas phase hydrates of trans-formanilide: A microscopic approach to the hydration sites of the peptide bond. The Journal of Physical Chemistry A 2001, 105, 969-973.

[7] Poblotzki, A.; Gottschalk, H. C.; Suhm, M. A. Tipping the scales: Spectroscopic tools for intermolecular energy balances. The Journal of Physical Chemistry Letters 2017, 8, $5656-5665$.

[8] Zimmermann, C.; Gottschalk, H.; Suhm, M. Three-dimensional docking of alcohols to ketones: an experimental benchmark based on acetophenone solvation energy balances. Physical Chemistry Chemical Physics 2020, 22, 2870-2877.

[9] Novick, S. E. Bibliography of rotational spectra of weakly bound complexes. 2019, https: //wesfiles.wesleyan.edu/home/snovick/SN_webpage/vdw.pdf.

[10] Caminati, W.; Grabow, J.-U. In Frontiers of molecular spectroscopy - Chapter 15, Section 4.2; Laane, J., Ed.; Elsevier, 2011.

[11] Pinacho, P.; Obenchain, D. A.; Schnell, M. New findings from old data: A semiexperimental value for the eQq of the nitrogen atom. The Journal of Chemical Physics 2020, 153, 234307.

[12] Potapov, A.; Asselin, P. High-resolution jet spectroscopy of weakly bound binary complexes involving water. International Reviews in Physical Chemistry 2014, 33, 275-300.

[13] Suhm, M.; Kollipost, F. Femtisecond single-mole infrared spectroscopy of molecular clusters. Physical Chemistry Chemical Physics 2013, 15, 10702-10721.

[14] Nedić, M.; Wassermann, T. N.; Larsen, R. W.; Suhm, M. A. A combined Raman- and infrared jet study of mixed methanol-water and ethanol-water clusters. Physical Chemistry Chemical Physics 2011, 13, 14050-14063.

[15] Gloaguen, E.; Mons, M.; Schwing, K.; Gerhards, M. Neutral peptides in the gas phase: conformation and aggregation issues. Chemical Reviews 2020, 120, 12490-12562.

[16] Zwier, T. S. The spectroscopy of solvation in hydrogen-bonded aromatic clusters. Annual Review of Physical Chemistry 1996, 47, 205-241. 
[17] Zischang, J.; Lee, J. J.; Suhm, M. A. Communication: Where does the first water molecule go in imidazole? The Journal of Chemical Physics 2011, 135, 061102.

[18] Maes, G.; Smets, J.; Adamowicz, L.; McCarthy, W.; Van Bael, M.; Houben, L.; Schoone, K. Correlations between ab initio and experimental data for isolated H-bonded complexes of water with nitrogen bases. Journal of Molecular Structure 1997, 410, 315322.

[19] Graton, J.; Berthelot, M.; Besseau, F.; Laurence, C. An enthalpic scale of hydrogen-bond basicity. 3. Ammonia, primary, secondary, and tertiary amines. The Journal of Organic Chemistry 2005, 70, 7892-7901.

[20] Chan, B.; Del Bene, J. E.; Elguero, J.; Radom, L. On the relationship between the preferred site of hydrogen bonding and protonation. The Journal of Physical Chemistry A 2005, 109, 5509-5517.

[21] Rozenberg, M.; Loewenschuss, A.; Marcus, Y. An empirical correlation between stretching vibration redshift and hydrogen bond length. Physical Chemistry Chemical Physics 2000, 2, 2699-2702.

[22] Plowright, R. J.; Gloaguen, E.; Mons, M. Compact folding of isolated four-residue neutral peptide chains: H-bonding patterns and entropy effects. ChemPhysChem 2011, 12, 18891899.

[23] Nasr, F. B.; Alata, I.; Scuderi, D.; Lepère, V.; Brenner, V.; Jaïdane, N.-E.; Zehnacker, A. Effects of complexation with sulfuric acid on the photodissociation of protonated Cinchona alkaloids in the gas phase. Physical Chemistry Chemical Physics 2019, 21, 15439-15451.

[24] Vogt, E.; Bertran Valls, P.; Kjaergaard, H. G. Accurate calculations of OH-stretching intensities with a reduced-dimensional local mode model including Eckart Axis embedding. The Journal of Physical Chemistry A 2020, 124, 932-942.

[25] Blodgett, K. N.; Fischer, J. L.; Zwier, T. S.; Sibert, E. L. The missing NH stretch fundamental in S 1 methyl anthranilate: IR-UV double resonance experiments and local mode theory. Physical Chemistry Chemical Physics 2020, 22, 14077-14087.

[26] Medel, R.; Suhm, M. A. Predicting OH stretching fundamental wavenumbers of alcohols for conformational assignment: Different correction patterns for density functional and wave-function-based methods. Physical Chemistry Chemical Physics 2021, 23, 5629-5643.

[27] Semrouni, D.; Sharma, A.; Dognon, J.-P.; Ohanessian, G.; Clavaguera, C. Finite temperature infrared spectra from polarizable molecular dynamics simulations. Journal of Chemical Theory and Computation 2014, 10, 3190-3199. 
[28] Miller, B. J.; Lane, J. R.; Kjaergaard, H. G. Intramolecular OH $\cdots \pi$ interactions in alkenols and alkynols. Physical Chemistry Chemical Physics 2011, 13, 14183-14193.

[29] Fu, W.; Hopkins, W. S. Applying machine learning to vibrational spectroscopy. The Journal of Physical Chemistry A 2018, 122, 167-171.

[30] Moult, J.; Fidelis, K.; Kryshtafovych, A.; Schwede, T.; Tramontano, A. Critical assessment of methods of protein structure prediction (CASP)-Round XII. Proteins: Structure, Function, and Bioinformatics 2018, 86, 7-15.

[31] Reilly, A. M.; Cooper, R. I.; Adjiman, C. S.; Bhattacharya, S.; Boese, A. D.; Brandenburg, J. G.; Bygrave, P. J.; Bylsma, R.; Campbell, J. E.; Car, R., et al. Report on the sixth blind test of organic crystal structure prediction methods. Acta Crystallographica Section B: Structural Science, Crystal Engineering and Materials 2016, 72, 439-459.

[32] Guthrie, J. P. A blind challenge for computational solvation free energies: introduction and overview. The Journal of Physical Chemistry B 2009, 113.

[33] Mobley, D. L.; Wymer, K. L.; Lim, N. M.; Guthrie, J. P. Blind prediction of solvation free energies from the SAMPL4 challenge. Journal of Computer-Aided Molecular Design 2014, 28, 135-150.

[34] Bannan, C. C.; Burley, K. H.; Chiu, M.; Shirts, M. R.; Gilson, M. K.; Mobley, D. L. Blind prediction of cyclohexane-water distribution coefficients from the SAMPL5 challenge. Journal of Computer-Aided Molecular Design 2016, 30, 927-944.

[35] Amezcua, M.; El Khoury, L.; Mobley, D. L. SAMPL7 Host-guest challenge overview: Assessing the reliability of polarizable and non-polarizable methods for binding free energy calculations. Journal of Computer-Aided Molecular Design 2021, 35, 1-35.

[36] Gottschalk, H. C.; Poblotzki, A.; Fatima, M.; Obenchain, D. A.; Pérez, C.; Antony, J.; Auer, A. A.; Baptista, L.; Benoit, D. M.; Bistoni, G., et al. The first microsolvation step for furans: New experiments and benchmarking strategies. The Journal of Chemical Physics 2020, 152, 164303.

[37] Gottschalk, H. C.; Poblotzki, A.; Suhm, M. A.; Al-Mogren, M. M.; Antony, J.; Auer, A. A.; Baptista, L.; Benoit, D. M.; Bistoni, G.; Bohle, F., et al. The furan microsolvation blind challenge for quantum chemical methods: First steps. The Journal of Chemical Physics 2018, $148,014301$.

[38] Benedict, W.; Plyler, E. K. Absorption spectra of water vapor and carbon dioxide in the region of 2.7 microns. Journal of Research of the National Bureau of Standards 1951, 46, $246-265$. 
[39] Bender, D. The Raman effect of water vapor. Physical Review 1935, 47, 252.

[40] QMbench - challenges for numerical quantum chemistry, https://qmbench.net/. 2021.

[41] Biswal, H. S.; Loquais, Y.; Tardivel, B.; Gloaguen, E.; Mons, M. Isolated monohydrates of a model peptide chain: Effect of a first water molecule on the secondary structure of a capped phenylalanine. Journal of the American Chemical Society 2011, 133, 3931-3942.

[42] Fischer, T. L.; Wagner, T.; Gottschalk, H. C.; Nejad, A.; Suhm, M. A. A rather universal vibrational resonance in 1:1 hydrates of carbonyl compounds. The Journal of Physical Chemistry Letters 2021, 12, 138-144.

[43] Chrayteh, M.; Burevschi, E.; Loru, D.; Huet, T. R.; Dréan, P.; Sanz, M. E. Disentangling the complex network of non-covalent interactions in fenchone hydrates via rotational spectroscopy and quantum chemistry, doi: 10.1039/d1cp02995a. Physical Chemistry Chemical Physics 2021,

[44] Bouchet, A.; Altnöder, J.; Broquier, M.; Zehnacker, A. IR-UV spectroscopy of jet-cooled 1-indanol: Restriction of the conformational space by hydration. Journal of Molecular Structure 2014, 1076, 344-351.

[45] Lei, J.; Zhang, J.; Feng, G.; Grabow, J.-U.; Gou, Q. Conformational preference determined by inequivalent n-pairs: Rotational studies on acetophenone and its monohydrate. Physical Chemistry Chemical Physics 2019, 21, 22888-22894.

[46] Spoerel, U.; Stahl, W. The aniline-water complex. Journal of Molecular Spectroscopy 1998, 190, 278-289.

[47] Melandri, S.; Maris, A.; Giuliano, B. M.; Caminati, W. Water-ketones hydrogen bonding: The rotational spectrum of cyclobutanone-water. The Journal of chemical physics 2005, 123, 164304.

[48] Bernhard, D.; Fatima, M.; Poblotzki, A.; Steber, A.; Pérez, C.; Suhm, M.; Schnell, M.; Gerhards, M. Dispersion-controlled docking preference: Multi-spectroscopic study on complexes of dibenzofuran with alcohols and water. Physical Chemistry Chemical Physics 2019, 21, 16032-16046.

[49] Gougoula, E.; Cole, D. J.; Walker, N. R. Bifunctional hydrogen bonding of imidazole with water explored by rotational spectroscopy and DFT calculations. The Journal of Physical Chemistry A 2020, 124, 2649-2659. 
[50] Zhang, X. K.; Lewars, E. G.; March, R. E.; Parnis, J. M. Vibrational spectrum of the acetone-water complex: A matrix isolation FTIR and theoretical study. The Journal of Physical Chemistry 1993, 97, 4320-4325.

[51] León, I.; Arnáiz, P.; Usabiaga, I.; Fernández, J. Mass resolved IR spectroscopy of anilinewater aggregates. Physical Chemistry Chemical Physics 2016, 18, 27336-27341.

[52] Brás, E. M.; Fischer, T. L.; Suhm, M. A. The hydrates of TEMPO: Water vibrations reveal radical microsolvation. Angewandte Chemie International Edition 2021, 60, 19013-19017.

[53] Broquier, M.; Lahmani, F.; Zehnacker-Rentien, A.; Brenner, V.; Millié, P.; Peremans, A. Hydrogen-bonded bridges in complexes of o-cyanophenol: Laser-induced fluorescence and IR/UV double-resonance studies. The Journal of Physical Chemistry A 2001, 105, 68416850 .

[54] Le Barbu, K.; Lahmani, F.; Mons, M.; Broquier, M.; Zehnacker, A. IR-UV investigation of the structure of the 1-phenylethanol chromophore and its hydrated complexes. Physical Chemistry Chemical Physics 2001, 3, 4684-4688.

[55] Venkatesan, V.; Fujii, A.; Ebata, T.; Mikami, N. A direct experimental evidence for an aromatic $\mathrm{C}-\mathrm{H} \cdots \mathrm{O}$ hydrogen bond by fluorescence-detected infrared spectroscopy. Chemical Physics Letters 2004, 394, 45-48.

[56] Van Bael, M. K.; Smets, J.; Schoone, K.; Houben, L.; McCarthy, W.; Adamowicz, L.; Nowak, M. J.; Maes, G. Matrix-isolation FTIR studies and theoretical calculations of hydrogen-bonded complexes of imidazole. A comparison between experimental results and different calculation methods. The Journal of Physical Chemistry A 1997, 101, 2397-2413.

[57] Burevschi, E.; Peña, I.; Sanz, M. E. Medium-sized rings: Conformational preferences in cyclooctanone driven by transannular repulsive interactions. Physical Chemistry Chemical Physics 2019, 21, 4331-4338.

[58] Burevschi, E.; Peña, I.; Sanz, M. E. Structures of complexes of cyclooctanone with water. 74th International Symposium on Molecular Spectroscopy. 2019.

[59] Vrolix, E.; Goethals, M.; Zeegers-Huyskens, T. Infrared study of hydrogen bond complexes involving 1, 3-dimethyl, 2-imidazolidinone and hydroxylic derivatives. Spectroscopy Letters 1993, 26, 497-507.

[60] Vigorito, A.; Paoloni, L.; Calabrese, C.; Evangelisti, L.; Favero, L. B.; Melandri, S.; Maris, A. Structure and dynamics of cyclic amides: The rotational spectrum of 1, 3dimethyl-2-imidazolidinone. Journal of Molecular Spectroscopy 2017, 342, 38-44. 
[61] Nelander, B. A matrix isolation study of the water-formaldehyde complex. The far-infrared region. Chemical Physics 1992, 159, 281-287.

[62] Lovas, F. J.; Lugez, C. The microwave spectrum and structure of CH2O-H2O. Journal of Molecular Spectroscopy 1996, 179, 320-323.

[63] Thomas, J.; Sukhorukov, O.; Jäger, W.; Xu, Y. Direct spectroscopic detection of the orientation of free $\mathrm{OH}$ groups in methyl lactate-(water) 1, 2 clusters: Hydration of a chiral hydroxy ester. Angewandte Chemie International Edition 2014, 53, 1156-1159.

[64] Katsyuba, S. A.; Spicher, S.; Gerasimova, T. P.; Grimme, S. Revisiting conformations of methyl lactate in water and methanol. The Journal of Chemical Physics 2021, 155, 024507.

[65] Galantay, E. 1-phenylcyclohexane-1,2-diols and their geometry. Tetrahedron 1963, 19, 319-321.

[66] Nieto, P.; Letzner, M.; Endres, T.; Schwaab, G.; Havenith, M. IR spectroscopy of pyridine-water structures in helium nanodroplets. Physical Chemistry Chemical Physics 2014, 16, 8384-8391.

[67] Mackenzie, R. B.; Dewberry, C. T.; Cornelius, R. D.; Smith, C.; Leopold, K. R. Multidimensional large amplitude dynamics in the pyridine-water complex. The Journal of Physical Chemistry A 2017, 121, 855-860.

[68] Melnik, D. G.; Gopalakrishnan, S.; Miller, T. A.; De Lucia, F. C. The absorption spectroscopy of the lowest pseudorotational states of tetrahydrofuran. The Journal of Chemical Physics 2003, 118, 3589-3599.

[69] Sahu, P. K.; Lee, S.-L. Hydrogen-bond interaction in 1:1 complexes of tetrahydrofuran with water, hydrogen fluoride, and ammonia: A theoretical study. The Journal of Chemical Physics 2005, 123, 044308.

[70] Sanz, M. E.; López, J. C.; Alonso, J. L.; Maris, A.; Favero, P. G.; Caminati, W. Conformation and stability of adducts of sulfurated cyclic compounds with water: Rotational spectrum of tetrahydrothiophene-water. The Journal of Physical Chemistry A 1999, 103, $5285-5290$.

[71] Lei, J.; Alessandrini, S.; Chen, J.; Zheng, Y.; Spada, L.; Gou, Q.; Puzzarini, C.; Barone, V. Rotational spectroscopy meets quantum chemistry for analyzing substituent effects on non-covalent interactions: The case of the trifluoroacetophenone-water complex. Molecules 2020, 25, 4899. 
[72] Heger, M.; Scharge, T.; Suhm, M. A. From hydrogen bond donor to acceptor: The effect of ethanol fluorination on the first solvating water molecule. Physical Chemistry Chemical Physics 2013, 15, 16065-16073.

[73] Thomas, J.; Xu, Y. Structure and tunneling dynamics in a model system of peptide co-solvents: Rotational spectroscopy of the 2,2,2-trifluoroethanol $\cdots$ water complex. The Journal of Chemical Physics 2014, 140,06B616_1.

[74] Ha, T. K.; Makarewicz, J.; Bauder, A. Ab initio study of the water-formaldehyde complex. The Journal of Physical Chemistry 1993, 97, 11415-11419.

[75] Dimitrova, Y.; Peyerimhoff, S. D. Theoretical study of hydrogen-bonded formaldehydewater complexes. The Journal of Physical Chemistry 1993, 97, 12731-12736.

[76] Ramelot, T. A.; Hu, C.-H.; Fowler, J. E.; DeLeeuw, B. J.; Schaefer, H. F. Theoretical study of hydrogen-bonded formaldehyde-water complexes. The Journal of Chemical Physics 1994, 100, 4347-4354.

[77] Nelander, B. Infrared spectrum of the water formaldehyde complex in solid argon and solid nitrogen. The Journal of Chemical Physics 1980, 72, 77-84.

[78] Shultz, M. J.; Vu, T. H. Hydrogen bonding between water and tetrahydrofuran relevant to clathrate formation. The Journal of Physical Chemistry B 2015, 119, 9167-9172.

[79] Meyer, R.; López, J. C.; Alonso, J. L.; Melandri, S.; Favero, P. G.; Caminati, W. Pseudorotation pathway and equilibrium structure from the rotational spectrum of jet-cooled tetrahydrofuran. The Journal of Chemical Physics 1999, 111, 7871-7880.

[80] Liu, X.; Sobolewski, A. L.; Borrelli, R.; Domcke, W. Computational investigation of the photoinduced homolytic dissociation of water in the pyridine--water complex. Physical Chemistry Chemical Physics 2013, 15, 5957-5966.

[81] Millen, D. J.; Mines, G. W. Hydrogen bonding in the gas phase. Part 5.--Infrared spectroscopic investigation of $\mathrm{O}-\mathrm{H} \cdots \mathrm{N}$ complexes formed by water: ammonia monohydrate and amine and pyridine monohydrates. Journal of the Chemical Society, Faraday Transactions 2: Molecular and Chemical Physics 1977, 73, 369-377.

[82] Choi, M. Y.; Miller, R. E. Infrared laser spectroscopy of imidazole complexes in helium nanodroplets: Monomer, dimer, and binary water complexes. The Journal of Physical Chemistry A 2006, 110, 9344-9351. 
[83] Juanes, M.; Saragi, R. T.; Pinacho, R.; Rubio, J. E.; Lesarri, A. Sulfur hydrogen bonding and internal dynamics in the monohydrates of thenyl mercaptan and thenyl alcohol. Physical Chemistry Chemical Physics 2020, 22, 12412-12421.

[84] Shahi, A.; Arunan, E. Microwave spectroscopic and theoretical investigations of the strongly hydrogen bonded hexafluoroisopropanol-..water complex. Physical Chemistry Chemical Physics 2015, 17, 24774-24782.

[85] Scharge, T.; Cézard, C.; Zielke, P.; Schütz, A.; Emmeluth, C.; Suhm, M. A. A peptide co-solvent under scrutiny: Self-aggregation of 2,2,2-trifluoroethanol. Physical Chemistry Chemical Physics 2007, 9, 4472-4490.

[86] Roccatano, D.; Colombo, G.; Fioroni, M.; Mark, A. E. Mechanism by which 2,2,2trifluoroethanol/water mixtures stabilize secondary-structure formation in peptides: A molecular dynamics study. Proceedings of the National Academy of Sciences 2002, 99, $12179-12184$.

[87] Borho, N.; Suhm, M. A.; Le Barbu-Debus, K.; Zehnacker, A. Intra- vs. intermolecular hydrogen bonding: dimers of alpha-hydroxyesters with methanol. Physical Chemistry Chemical Physics 2006, 8, 4449-4460.

[88] Merten, C.; Xu, Y. Chirality transfer in a methyl lactate-ammonia complex observed by matrix-isolation vibrational circular dichroism spectroscopy. Angewandte Chemie 2013, 125, 2127-2130.

[89] Gnanasekar, S. P.; Arunan, E. Structure and internal motions of a multifunctional alcoholwater complex: rotational spectroscopy of the propargyl alcohol $\cdots$ H2O Dimer. The Journal of Physical Chemistry A 2021,

[90] Juanes, M.; Lesarri, A.; Pinacho, R.; Charro, E.; Rubio, J. E.; Enríquez, L.; Jaraíz, M. Sulfur hydrogen bonding in isolated monohydrates: Furfuryl mercaptan versus furfuryl alcohol. Chemistry-A European Journal 2018, 24, 6564-6571.

[91] Lovejoy, C. M.; Nesbitt, D. J. Slit pulsed valve for generation of long-path-length supersonic expansions. Review of Scientific Instruments 1987, 58, 807-811.

[92] Campargue, R. Progress in overexpanded supersonic jets and skimmed molecular beams in free-jet zones of silence. The Journal of Physical Chemistry 1984, 88, 4466-4474.

[93] Altnoeder, J.; Krueger, K.; Borodin, D.; Reuter, L.; Rohleder, D.; Hecker, F.; Schulz, R. A.; Nguyen, X. T.; Preiss, H.; Eckhoff, M., et al. The guinness molecules for the carbohydrate formula. The Chemical Record 2014, 14, 1116-1133. 
[94] Simons, J. Good vibrations: probing biomolecular structure and interactions through spectroscopy in the gas phase. Molecular Physics 2009, 10\%, 2435-2458.

[95] Bakels, S.; Gaigeot, M.-P.; Rijs, A. M. Gas-phase infrared spectroscopy of neutral peptides: Insights from the far-IR and THz domain. Chemical Reviews 2020, 120, 3233-3260.

[96] Nakamura, T.; Schmies, M.; Patzer, A.; Miyazaki, M.; Ishiuchi, S.-i.; Weiler, M.; Dopfer, O.; Fujii, M. Solvent migration in microhydrated aromatic aggregates: Ionizationinduced site switching in the 4-aminobenzonitrile-water cluster. Chemistry-A European Journal 2014, 20, 2031-2039.

[97] Auty, A. R.; Jones, A. C.; Phillips, D. Fluorescence excitation spectra and decay times of jet-cooled dibenzofuran and the dibenzofuran-water complex. Chemical Physics Letters 1984, 112, 529-533.

[98] Piani, G.; Pasquini, M.; López-Tocón, I.; Pietraperzia, G.; Becucci, M.; Castellucci, E. The aniline-water and aniline-methanol complexes in the S1 excited state. Chemical Physics 2006, 330, 138-145.

[99] Gottschalk, H. C.; Fischer, T. L.; Meyer, V.; Hildebrandt, R.; Schmitt, U.; Suhm, M. A. A sustainable slit jet FTIR spectrometer for hydrate complexes and beyond. Instruments 2021, 5, 12 .

[100] Atomic weights and isotopic compositions for all elements, https://physics.nist.gov/ cgi-bin/Compositions/stand_alone.pl.

[101] Ben Faleh, A.; Warnke, S.; Rizzo, T. R. Combining ultrahigh-resolution ion-mobility spectrometry with cryogenic infrared spectroscopy for the analysis of glycan mixtures. Analytical Chemistry 2019, 91, 4876-4882.

[102] Spieler, S.; Duong, C. H.; Kaiser, A.; Duensing, F.; Geistlinger, K.; Fischer, M.; Yang, N.; Kumar, S. S.; Johnson, M. A.; Wester, R. Vibrational predissociation spectroscopy of cold protonated tryptophan with different messenger tags. The Journal of Physical Chemistry A 2018, 122, 8037-8046.

[103] Fischer, K. C.; Sherman, S. L.; Garand, E. Competition between solvation and intramolecular hydrogen-bonding in microsolvated protonated glycine and $\beta$-alanine. The Journal of Physical Chemistry A 2020, 124, 1593-1602.

[104] Caminati, W.; López, J. C.; Blanco, S.; Mata, S.; Alonso, J. L. How water links to cis and trans peptidic groups: the rotational spectrum of N-methylformamide-water. Physical Chemistry Chemical Physics 2010, 12, 10230-10234. 
[105] Caminati, W.; Favero, L. B.; Favero, P. G.; Maris, A.; Melandri, S. Intermolecular hydrogen bonding between water and pyrazine. Angewandte Chemie International Edition 1998, 37, 792-795.

[106] Li, X.; Lu, T.; Obenchain, D. A.; Zhang, J.; Herbers, S.; Grabow, J.-U.; Feng, G. The characteristics of disulfide-centered hydrogen bonds. Angewandte Chemie International Edition 2021, 60, 5838-5842.

[107] Du, L.; Tang, S.; Hansen, A. S.; Frandsen, B. N.; Maroun, Z.; Kjaergaard, H. G. Subtle differences in the hydrogen bonding of alcohol to divalent oxygen and sulfur. Chemical Physics Letters 2017, 667, 146-153.

[108] Leopold, K. R. Hydrated acid clusters. Annual Review of Physical Chemistry 2011, 62, 327-349.

[109] Schnitzler, E. G.; Seifert, N. A.; Ghosh, S.; Thomas, J.; Xu, Y.; Jäger, W. Hydration of the simplest $\alpha$-keto acid: A rotational spectroscopic and ab initio study of the pyruvic acid-water complex. Physical Chemistry Chemical Physics 2017, 19, 4440-4446.

[110] Kryshtafovych, A.; Schwede, T.; Topf, M.; Fidelis, K.; Moult, J. Critical assessment of methods of protein structure prediction (CASP)-Round XIII. Proteins: Structure, Function, and Bioinformatics 2019, 87, 1011-1020.

[111] Groom, C. R.; Reilly, A. M. Sixth blind test of organic crystal-structure prediction methods. Acta Crystallographica Section B: Structural Science, Crystal Engineering and Materials 2014, 70, 776-777.

[112] Kjaersgaard, A.; Vogt, E.; Hansen, A. S.; Kjaergaard, H. G. Room temperature gas-phase detection and Gibbs energies of water amine bimolecular complex formation. The Journal of Physical Chemistry A 2020, 124, 7113-7122. 